\title{
Development of high-efficiency, highly-diffused prismatic panel and its application ${ }^{\dagger}$
}

\author{
Koji SATO (Member) \\ (Toshiba Lighting \& Technology Corp. Lamp \& Lighting Laboratory) \\ Nobuo MATSUSHITA (Member)* \\ (Toshiba Lighting \& Technology Corp. Institution \& Industrial Lighting Division)
}

\begin{abstract}
This paper describes the developmental process of new prismatic panel for lighting fixture which satisfies both high efficiency and diffusion ability. Concretely, the result of improved opal panel for high efficiency and diffusion ability, the theory of accomplishing high diffusion ability by prismatic panel, the process of selecting prismatic element, the simulation of luminance of prismatic panel by inverse-ray tracing method and the specification of surface-mounted fluorescent lighting fixture applied with this prismatic panel are reported.

The prismatic element is lozenge-shaped, with a length of $5.0 \mathrm{~mm}$, a width of $2.9 \mathrm{~mm}$ and a height of $2.2 \mathrm{~mm}$. The light which goes to the prismatic element just under the lamp is totally reflected at the internal prismatic surface, consequently apparent luminance of lamp through the panel is reduced. While the light which goes to the prismatic element between lamps is refracted downward, so luminance of panel between lamps is increased. Applying this prismatic panel, very thin surface-mounted fluorescent fixture has been developed.
\end{abstract}

\section{Introduction}

In the recent commercial facilities, a lighting has taken a great role in order to let the goodsfor-sales show effectively. Especially, as a general-lighting fixture in the store, a simple and compact one is desirable in order to increase a degree of freedom of commercial space and from the standpoint of ceiling design, thereby the study of small-sizing and thinning the fixture by using a compact light source has been carried out energeticly.

While a lighting fixture provided with straighttube fluorescent lamp is extensively used as a general-lighting fixture, a desire for thinning the fixture is also strong as well. The currentlyused surface mounted fluorescent lighting fixture with cover, which is favorably used in the store where a soft atmosphere with controlled glare is required, has a difficulty in thinning the fixture because a diffusion ability

$\dagger$ This paper is translated from Journal of IEI-J Vol. 73, No. 2 for which IEI-J prize was awarded.

* 1-201-1 Hunakoshi-cho, Yokosukashi 237 of prismatic panel has a limitation and a lamp image or a shadow of ballast appears distinctly.

By means of optical design technique, the authers have developed a power-saving type display lamp for road signboard," a HID downlight provided with prismatic panel for attaining a squared illuminance distribution, ${ }^{2)}$ a floodlight provided with an asymmetric reflecting mirror having both high coefficient of utilization and uniformity and a high efficiency floodlight utilizing vapor-deposited reflecting mirror, etc., so far. Each of them is a result obtained by optimizing the shape of optical element such as lens, prism and reflecting mirror by means of optical design technique using a computer so as to obtain an optimum luminous intensity distribution depending on each of various applications. As an optical design method, the authers use two different methods, i.e., a ray tracing method, generally used, and an inverse-ray tracing method in which a calculation is carried out along an inverse optical path from an illuminated point to a light source through a reflecting mirror or prism. ${ }^{3 \text { ) }}$ 
Now, by applying this inverse-ray tracing method for performing a luminous intensity distribution control to a luminance control member for making the luminance of panel surface uniform, a new type of prismatic panel could be developed, thereby being able to solve the problem of lamp image usually generated by thinning the fixture. In this paper, the results of experiment for improving the opal panel which was performed as an approach to a highefficiency, highly-diffused panel and its limitation, the theory of accomplishing a high diffusion ability by prismatic panel, the process of selecting prismatic element, the simulation of luminance of panel surface by inverse-ray tracing method and the specification of lighting fixture applied with this new prismatic panel will be reported hereinafter.

\section{Discussion on High-Efficiency, High- Diffused Panel}

The necessary condition of a panel with high diffusion ability used in a lighting fixture is to transmit and diffuse the light sufficiently. By thinning the fixture, the luminance at the panel portion just under the lamp will be high due to a shortened distance between lamp and panel and the luminance of the panel portion intermediate between the lamps will be low. As a result, a light and darkness generally called as "lamp image" will be generated on the panel surface. It is said that it is difficult by the conventional opal panel or prismatic panel to accomplish a high efficiency while a sufficient diffusion ability is maintained without light and darkness. Therefore, in order to solve such a problem, the authers started a study of a new panel provided with both a high diffusion ability of opal panel and high efficiency of prismatic panel. First of all, a study was started from accomplishing a high transmittance of opal panel as an approach thereto and, then, a study was continued so as to accomplish a high diffusion ability of prismatic panel.

\subsection{Accomplishing a High Transmittance of Opal Panel}

An opal panel is produced generally by adding a white pigment into a transmission material such as polystyrene or acrylic resin. Consequently, it is expectable that the transmittance and diffusion ability of panel will depend on the content, type and particle diameter of white pigment. Therefore, an evaluation experiment was carried out by using test samples in order to investigate the dependance of the transmittance and luminance ratio of opal panel on the content and particle diameter of white pigment. The sample was manufactured by using polystyrene resin with a thickness of $2 \mathrm{~mm}$ as a substrate material and calcium carbonate with a particle diameter of $2.5-5.5 \mu \mathrm{m}$ and a content of $0.5-4.0 \mathrm{wt} \%$ as a white pigment. For the measurement of the transmittance of the test sample, a double integral sphere type transmittance meter was used so as to make the light incident from all directions by taking into consideration the opal panel for fluorescent lighting fixture. Furthermore, for the measurement of luminance ratio, the distance between lamp and panel was approximated to the currently-used surface mounted fluorescent lighting fixture with cover. Fig. 1 shows these measurement conditions.

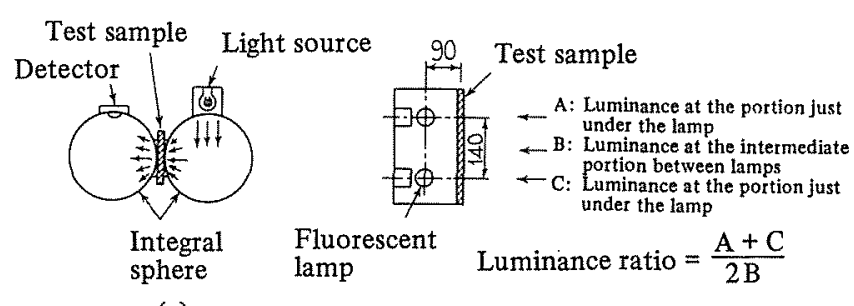

(a)

(b)

Fig: 1. Measurement conditions of transmittance of opal panel and luminance ratio

(a) Measurement of transmittance

(b) Measurement of luminance ratio

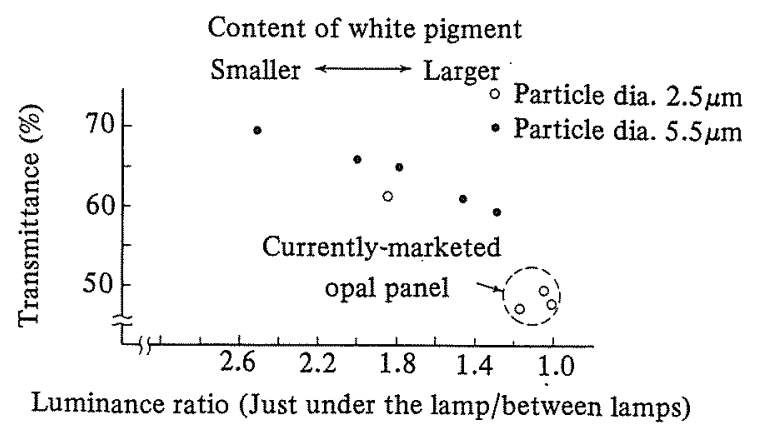

Fig. 2. Transmittance and luminance ratio of test opal panel 
Fig. 2 shows an example of measurement results of test sample on transmittance and luminance ratio. While the transmittance decreases in accordance with the addition of white pigment, the luminance ratio was improved. It was found that the larger the particle diameter of white pigment, the more the luminance ratio was improved at the same transmittance. However, it was difficult to obtain both of satisfactory diffusion ability and high transmittance, i.e., to attain a sample with a high transmittance of $60 \%$ or more while maintaining a diffusion ability of the currently-used commercial opal panel (luminance ratio of 1.0-1.3) as shown in Fig. 2.

\subsection{Accomplishing a High Diffusion Ability of Prismatic Panel}

Prismatic panel is usually manufactured by forming a transparent material so as to have a rough surface for diffusing the light. For that reason, a large number of prismatic panel has a larger transmittance than opal panel. Consequently, before starting the study, a target was placed on the reduction of lamp image while maintaining a high transmittance of prismatic panel. Instead of diffusing the light irregularly by adding a white pigment or roughening the panel surface, a positive control of light by a precise optical design was taken into consideration as a means for increasing diffusion ability. Practically, a study was started based on the theory that if an apparent luminance through a panel could be reduced by effectively refracting or totally reflecting the light to be incident into the panel portion just under the lamp where the lamp image would be generated, it would be capable of accomplishing both a high transmittance and a satisfactory luminance ratio simultaneously as a result of an improved uniformity of luminance.

\section{Development of High-Efficiency, Highly- Diffused Prismatic Panel}

\subsection{Discussion on Prismatic Element}

When a light is controlled by using prismatic panel, there would be imagined two methods, i.e., a method for obtaining squared illuminance distribution $^{4)}$ or twin beam luminous intensity distribution ${ }^{5}$ by particularly determining the positions of light source and panel and a method for controlling the luminous intensity distribution by using a prismatic panel comprising repetitively layouted prismatic elements with the same shape.6)-9) The particular determination of the positions of light source and lamp would limit the number of lamps or the fixture design in a great degree due to a standardized fixture. Therefore, in this development, a target was placed decisively on the development of a general-purpose high-efficiency, highlydiffused prismatic panel so that a stereoscopic shape of each prismatic element was studied one by one which was hardly discussed so far.

Table 1 Study of basic shape of prism

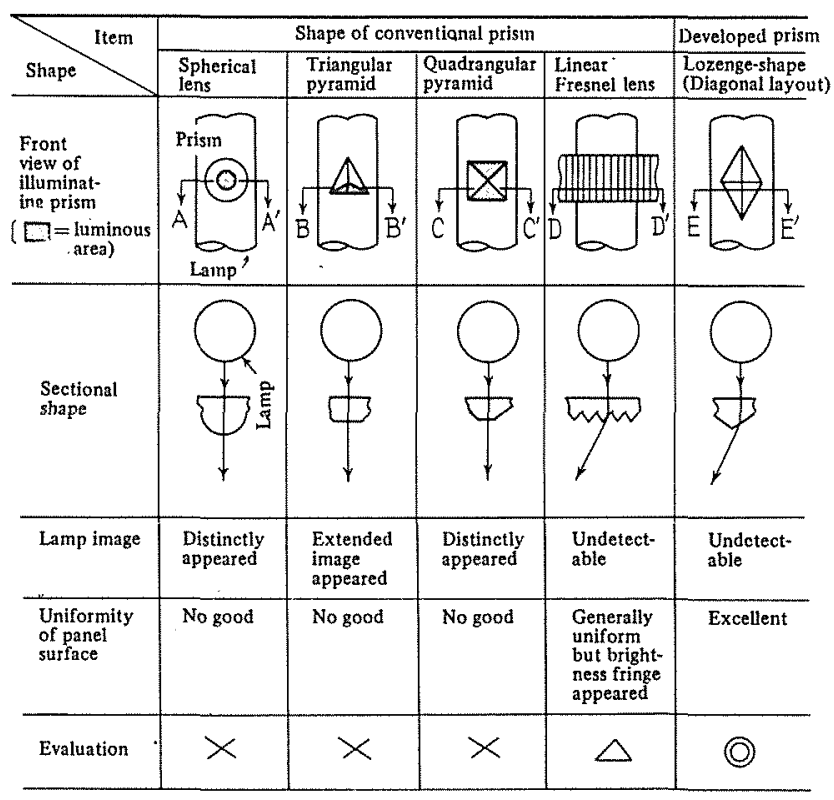

Table 1 diagrammatically shows how the light incident into the panel surface just under the lamp is controlled converning a variety of actually practicable elements, wherein the diffusion ability was evaluated qualitatively by a computer simulation. As shown in the sectional shape of Table 1, the spherical lens or triangular prism can not control the light in the crosssection orthogonal to the center line of the lamp if the cross-section of the element is orthogonal to the direct light from the lamp, thereby a lamp image being generated distinctly. It is 
understood from this point that the spherical lens, triangular pyramid and quadrangular pyramid can not make the panel surface bright uniformly. Furthermore, it is expectable that the Fresnel lens will easily generate brightness fringes in the longitudinal direction. From the above discussion, it was discovered that a prism element comprising the lozenge-shaped prism arranged diagonally is promising.

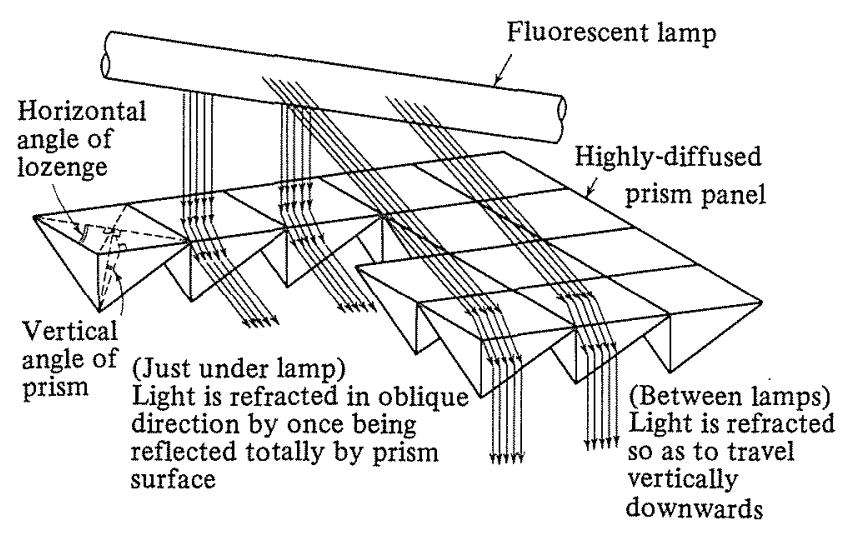

Fig. 3. Optical principle of highly-diffused prism
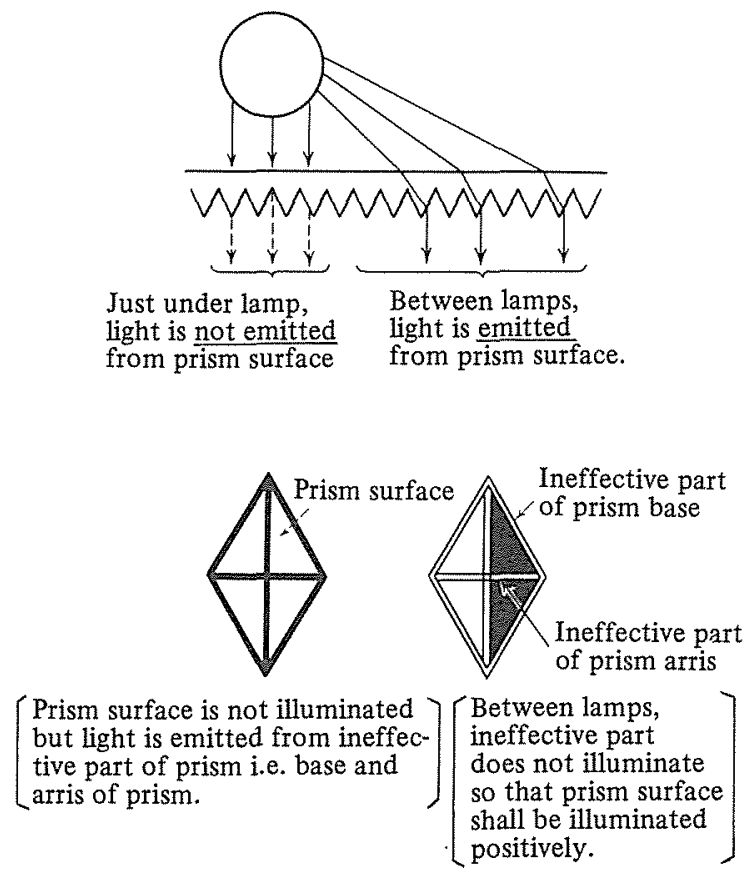

Fig. 4. Operation of accomplishing high diffusion ability by prism control

\subsection{Basic Operation of Lozenge-Shaped Prism}

Fig. 3 shows an optical principle of lozengeshaped prism. A lozenge-shaped prism having a basic shape comprising a quadrangular pyramid prism with a lozenge-shaped base refracts the light travelling vertically downwards towards an oblique direction by an operation of prism of total reflection and refracts the light travelling for the intermediate portion between lamps vertically downwards.

Then, Fig. 4 diagrammatically shows the operation of prism for accomplishing a uniform luminance of panel surface, i.e., a high diffusion ability. When the prism element is observed in detail, the element is divided into an effective part formed as a prism surface and an ineffective part innevitably produced for forming a panel. As shown in Fig. 4, at the portion just under the lamp the ineffective part is illuminating and at the intermediate portion between lamps the effective part is illuminating. Therefore, it was assumed that a uniform luminance of total panel surface would be accomplished by balancing the area of both parts so as to be equal.

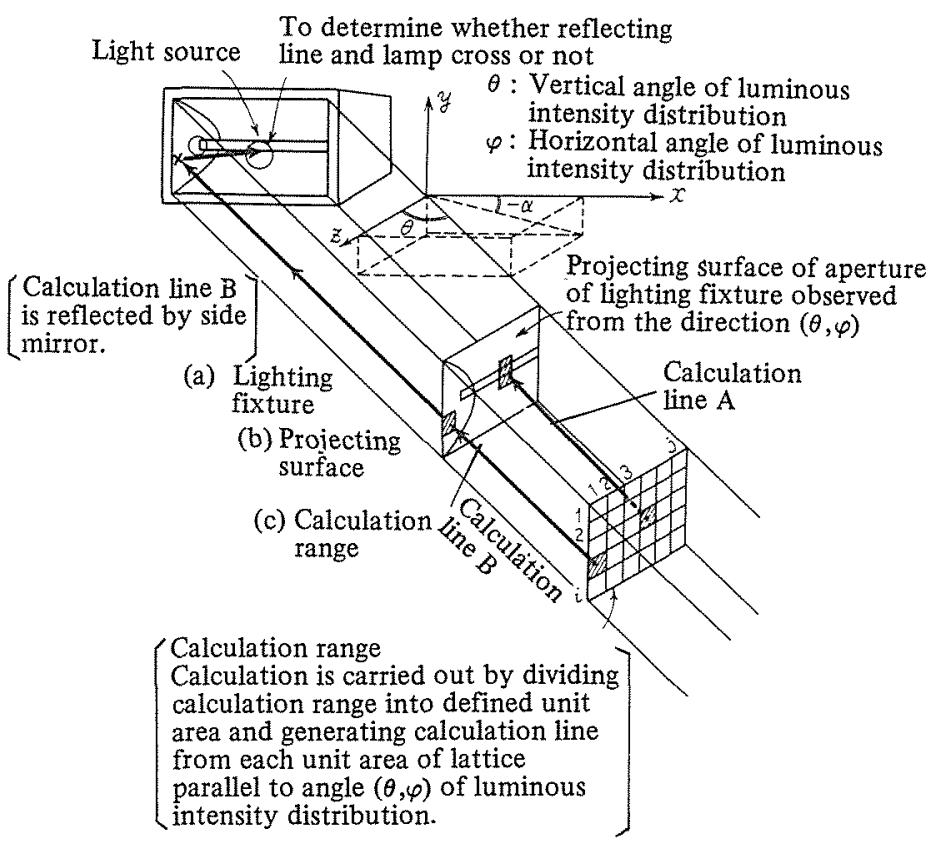

Fig. 5. Basic principle of inverse-ray tracing method 


\subsection{Optical Design of Lozenge-Shaped Prism}

While in the previous chapter an operation of lozenge-shaped prism was explained qualitatively, in this chapter an optical design of lozenge-shaped prism for making the luminance of the prism surface uniform will be reported in detail. At first, a theory of inverse-ray tracing method used in the optical design of prism will be explained and subsequently a design method of prism will be described.

\subsubsection{Outline of Inverse-Ray Tracing Method}

When an optical system of lighting fixture is designed, the optical system is observed, usually, concerning what part of the optical system is illuminating while evaluating its optical system or measuring the luminous intensity distribution or luminance. If a desired performance could not be obtained, a decision will be given concerning what part of the optical system shall be modified and how modified on the basis of the observation results of the luminance of the optical system. If the decision of shape modification is given by computer processing, an optical system approximated more closely to a desired target could be developed at a design stage. It is inverse-ray tracing method that was invented from such a theory. It can be applied to a basic optical system such as reflector, lens and prism as well as to buffle, louver, etc.

Fig. 5 diagrammatically shows a method for obtaining a luminous intensity in the directions of vertical angle $\theta^{\circ}$ and horizontal angle $\psi^{\circ}$ by applying the inverse-ray tracing method to an optical system provided with reflecting mirror. If the quadrilateral reflector is projected in the direction of $(\theta, \psi)$, a profile as shown in Fig. 5 will be obtained. Then, the projected profile divided into a plurality of divisions of equal area for producing a lattice as shown in Fig. 5. A "calculation line" is generated from the center of each division of the lattice. It is to say that this "calculation line" represents a so-called sight line of an observer. The calculation line A shown in Fig. 5 hits the light source so that it is determined as a direct light and the calculation line $\mathrm{B}$ hits the side reflector and then hits the light source by a reflection so that it is determined as a primary reflecting light. From such a determination of each calculation line departing from lattice, a luminous area can be obtained. By multiplying the luminous area by luminance, a luminous intensity can be obtained. The luminance in this case is equal to the product of the luminance of light source with the reflectance according to the rule of invariable luminance. ${ }^{10}$ ) Although the inverse-ray tracing method was explained by an example of reflector, this method can be applied to a transparent optical system such as prism and lens in the same way.

When the inverse-ray tracing method is applied to the optical design of lighting fixture, the following features will be obtained. ${ }^{11}$ )

(1) By taking a correspondence of luminous intensity with the luminous area, it is easy to modify the optical system or to make a feedback for improvement.

(2) It is easy to evaluate the effect of the shielding articles at the emission side such as the trim or buffle of the fixture.

(3) The volume of calculation is reduced compared to the prior ray tracing method.

The optical design technique applying this inverse-ray tracing method has been applied to the development of lens," prism ${ }^{2)}$ and reflector. ${ }^{3)}$ This time, a new program for checking the luminous pattern of each prism surface of lozenge-shaped prism was developed.

\subsubsection{Design of Lozenge-Shaped Prism}

Fig. 6 shows a flowchart of the program for simulating the optical property of lozengeshaped prism by using the inverse-ray tracing method. By inputting the data of light source and prism shape, it is capable of determining which prism is luminous. In a practical design, the luminous pattern of each prism surface was simulated by changing the horizontal angle $(\alpha)$, as shown in Fig. 7 , from $15^{\circ}$ to $75^{\circ}$ by an increment of $15^{\circ}$, the vertical angle $(\beta)$ from $20^{\circ}$ to $60^{\circ}$ by an increment of $5^{\circ}$ and the distance between the center of lamp and the internal surface of panel from $20 \mathrm{~mm}$ to $40 \mathrm{~mm}$ by an increment of $5 \mathrm{~mm}$.

Fig. 7 shows a simulation result of the luminous pattern of panel surface observed from the location just below by changing the horizontal angle of lozenge-shaped prism at $30^{\circ}$ or $15^{\circ}$ and the vertical angle of the prism at $30^{\circ}$ or 


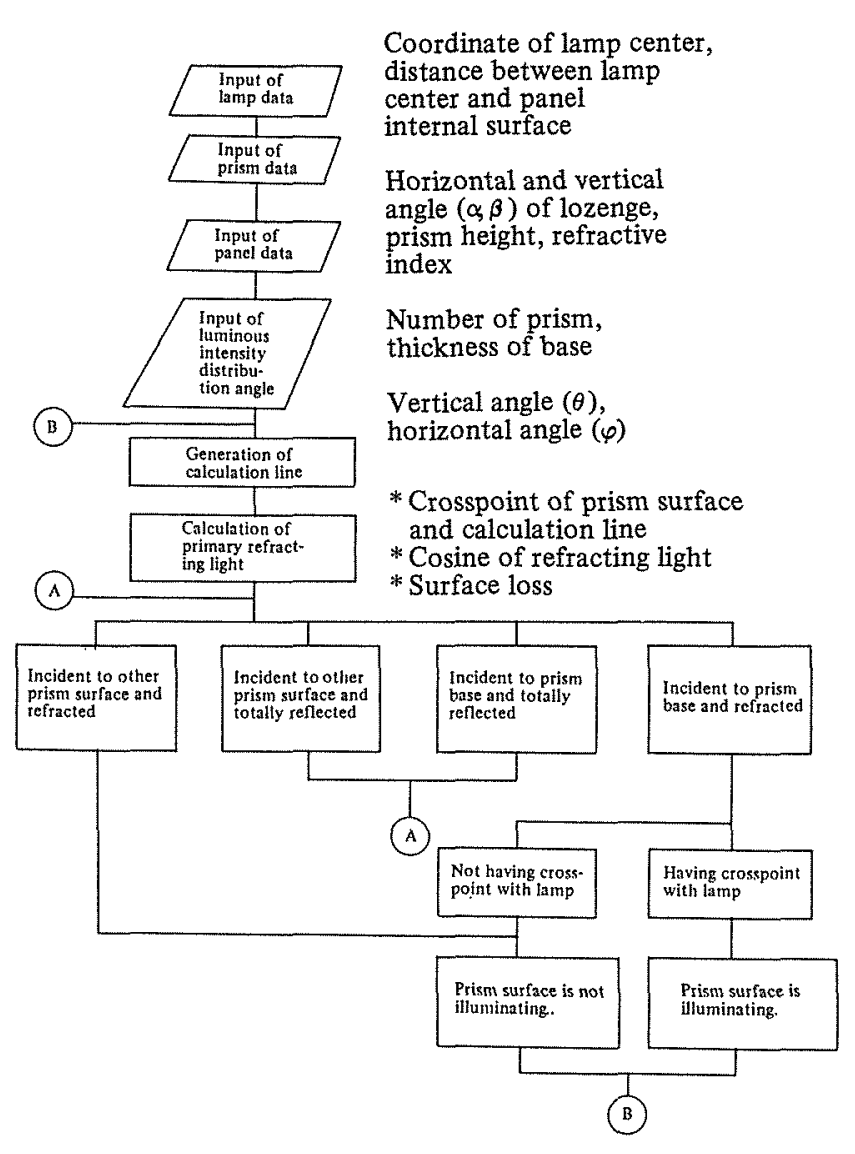

Fig. 6. Flowchart of program for simulating optical property of lozengeshaped prism
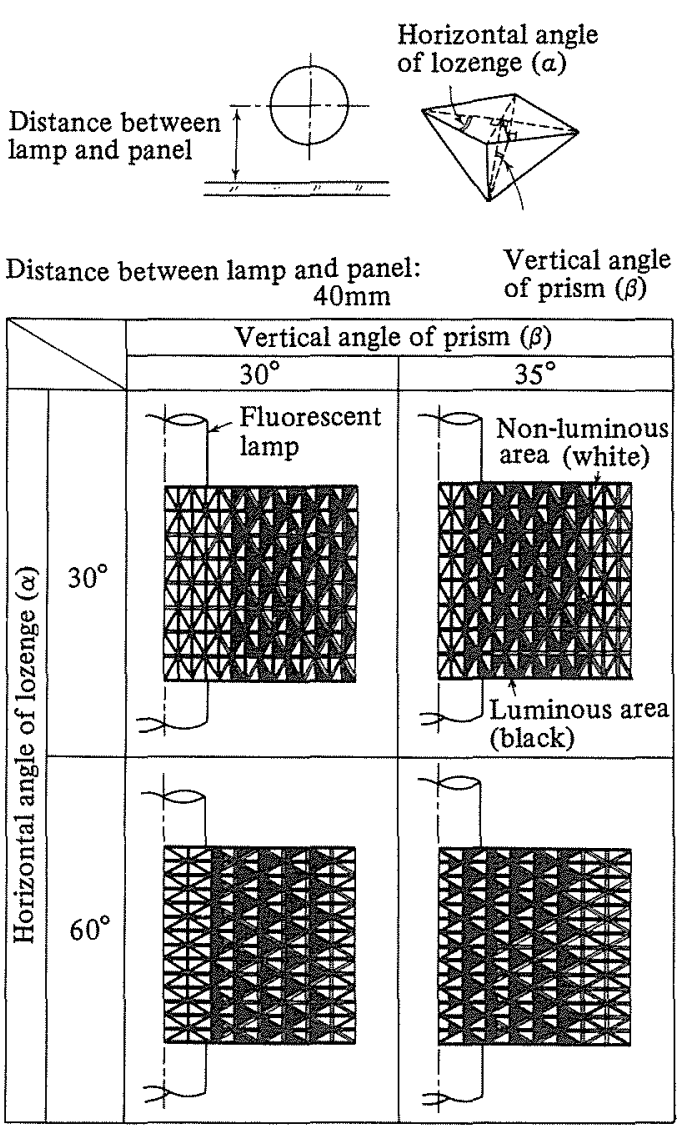

Fig. 7. Simulation result of luminous pattern of panel surface (Black area shows luminous state.)

\subsection{Forming of Lozenge-Shaped Prism Panel}

In order to be used extensively as a prism panel for lighting fixture, it is required that the prism panel can correspond not only to an optical performance but also to a variety of fixture types from 2-lamp type to multi-lamp type and also practically the prism panel must be supplied stably in an industrial level. Table 2 shows a comparison of various production methods of prism panel. In the table, the methods of roll forming, thermal press forming and injection forming, which are assumed to be appropriate for the production of the prismpanel at present, are compared including the above-mentioned problems. It was concluded that the roll forming method was most appropriate for forming the lozenge-shaped prism because it has many of advantages such as being correspondable to a multi-lamp type, being capable of supplying a large-size and being small in the mold investment, while it is slightly poor in the accuracy of the shape. 
Table 2 Comparison of forming method

\begin{tabular}{l|l|l|l|l}
\hline $\begin{array}{l}\text { Forming } \\
\text { method }\end{array}$ & $\begin{array}{c}\text { Evaluation } \\
\text { item } \\
\text { sional } \\
\text { freedom }\end{array}$ & $\begin{array}{c}\text { Produc- } \\
\text { tivity }\end{array}$ & $\begin{array}{c}\text { Shape } \\
\text { accuracy }\end{array}$ & $\begin{array}{c}\text { Mold } \\
\text { invest- } \\
\text { ment }\end{array}$ \\
\hline Roll forming & Large & High & Good & Middle \\
\hline $\begin{array}{l}\text { Thermal press } \\
\text { forming }\end{array}$ & Small & Low & Excellent & Small \\
\hline Injection forming & Small & High & $\begin{array}{l}\text { Very } \\
\text { excellent }\end{array}$ & Large \\
\hline
\end{tabular}

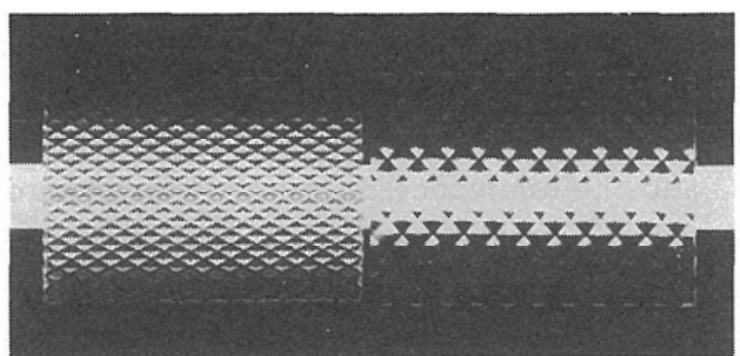

$\begin{aligned} & \text { Lozenge-shaped } \\ & \text { prism }\end{aligned}$
(Developed) $\quad\left(\begin{array}{l}\text { Conventional } \\ \text { Quadrangular prism } \\ \text { with } 5 \mathrm{~mm} \text { square and } \\ 2.8 \mathrm{~mm} \text { thickness }\end{array}\right)$

Distance between lamp and panel: $40 \mathrm{~mm}$

Fig. 8. Luminous pattern of prism surface

Fig. 8 compares the luminous patterns of the lozenge-shaped prism produced by the roll forming method and of the conventional prism. In the case of lozenge-shaped prism, both horizontal and vertical angle were $30^{\circ}$ and the dimension of lozenge was determined from the forming condition such that the length, width and height of prism were $5 \mathrm{~mm} .2 .9 \mathrm{~mm}$ and $2.2 \mathrm{~mm}$, respectively. Compared to the conventional prism, in the lozenge-shaped prism the portion outside of the location just under the lamp is also illuminating more distinctly, thereby it can be appreciated that the total panel surface is illuminating uniformly in the same way as a simulation result shown in Fig. 7 .

\section{Application to Thinned, Surface Mounted Fluorescent Lighting Fixture}

The lozenge-shaped prism developed this time was used as a panel for the surface mounted lighting fixture with $2 \times 40 \mathrm{~W}$ straight-tube fluorescent lamp and the luminance ratio of the panel surface was measured. The fixture used for the experiment has a height of $72 \mathrm{~mm}$, a lamp pitch of $140 \mathrm{~mm}$ and a distance of $40 \mathrm{~mm}$ between the center of lamp and the internal surface of panel. For the measurement of luminance ratio, a range of about $16 \mathrm{~mm}$ corresponding to the central portion of lamp and the intermediate portion between lamps was measured from the front of the fixture by a luminance meter with a field angle of $1^{\circ}$.

Fig. 9 graphically shows the measurement result of this luminance ratio and fixture efficiency and also the measurement result of the conventional quadrangular pyramid panel and opal panel together. The lozenge-shaped prism panel obtained the same luminaire efficiency $(65 \%)$ as the conventional prism panel and a uniformity of luminance (luminance ratio $=1.4$ ) comparable to the opal panel. When the ficture height was reduced furthermore, the lamp image appeared distinctly. Therefore, it was concluded that the fixture height of $72 \mathrm{~mm}$ was optimum also from the point of ballast shadow described later.

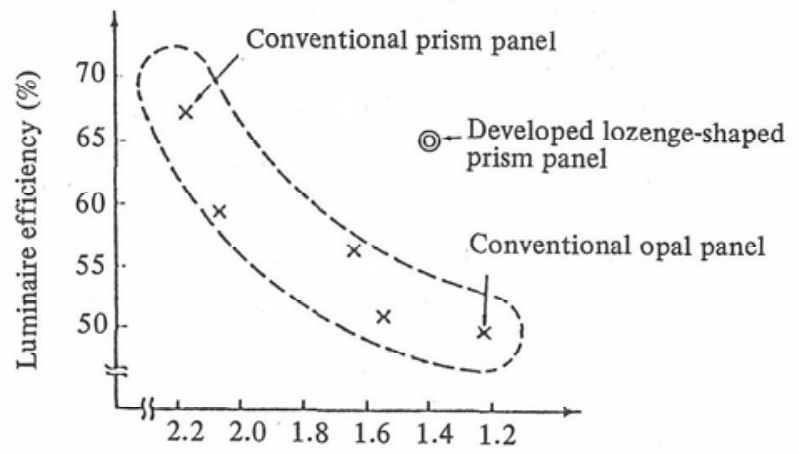

Luminance ratio of panel surface (Just under lamp/between lamps)*

Fixture: surface mounted lighting fixture with $2 \mathrm{X} 40 \mathrm{~W}$ fluorescent lamp (fixture height: $72 \mathrm{~mm}$ )

* Fixture shown in Fig. 10 was used. It was measured from $1 \mathrm{~m}$ distance by luminance meter with field angle of $1^{\circ}$.

Fig. 9. Relation between luminaire efficiency and luminance ratio of panel surface

2. Ballast 3. Lamp socket

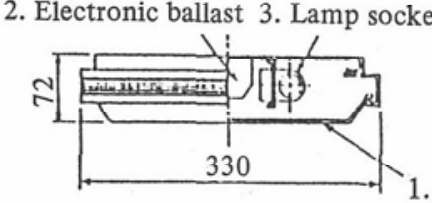

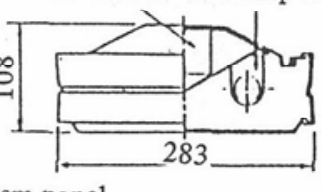

. Prism panel (for 2 X 40W lamp)

Conventional fixture
Fig. 10. Comparison of fixture height of surface mounted fluorescent lighting fixture 


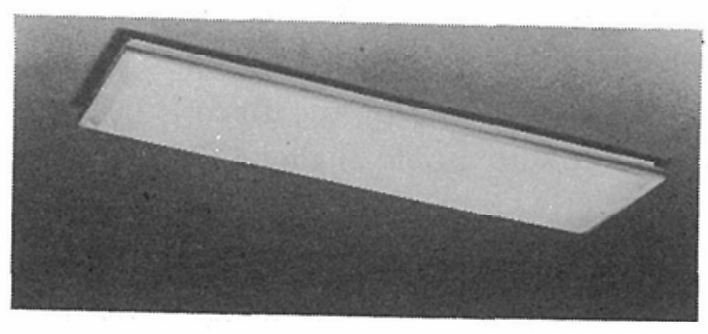

Fig. $112 X 40 \mathrm{~W}$ thinned, surface mounted fluorescent lighting fixture (aluminum trim)

Fig. 10 shows the cross-section of the thinned, surface mounted fluorescent lighting fixture and the conventional surface mounted fluorescent lighting fixture. In order to maintain a uniformity of luminance of panel surface, it is necessary to replace the conventional $\mathrm{Cu}-\mathrm{Fe}$ type ballast with thin-type Electronic ballast. Fig. 11 shows an outlook of surface mounted fluorescent lighting fixture.

As described in the above, this fixture is appropriate for being used in a small space such as meeting room, store, in particular, specialty store and housing, etc., in which a visibility of panel surface viewed upwards from the floor is concerned.

\section{Conclusion}

The process of improving a uniformity of luminance of panel for the fluorescent lighting fixture was explained. At first, an experiment was tried for improving the opal panel but it did not succeed in obtaining a high transmittance, while maintaining a diffusion ability, by a method for changing a type of white pigment. Then, by applying the inverse-ray tracing method, which was developed by authers, to a luminance control technique for making the luminance of prism panel uniform, the followings were obtained.

(1) It was discovered by reviewing the shape of prism element that the lozenge-shaped prism was appropriate for controlling the light travelling downwards just under lamp.

(2) It was found that the luminance downwards just under the panel can be made uniform by controlling the luminous pattern comprised of 2 parts, i.e., an effective part which can control the light as a prism surface and an ineffec- tive part which is produced inevitably for forming a panel.

(3) It was discovered by a prism evaluation program applied with the inverse-ray tracing method that the lozenge-shaped prism with both horizontal and vertical angle of $30^{\circ}$ was most appropriate.

(4) It was found that by applying the lozenge-shaped prism to the thinned, surface mounted fluorescent lighting fixture with a height of $72 \mathrm{~mm}$ an almost satisfactory optical performance, i.e., the luminaire efficiency of $65 \%$ and the luminance ratio of 1.4 downwards just under the panel surface could be obtained.

As a future task, it is required to investigate the prism which has a uniformity not only in the front direction of the fixture but also in all directions.

References (all in Japanese)

(1) Sato, Okada, Nakase, Muraki: Simulation of Luminous Intensity Distribution in Lens Optical System and its Application, Preprints of Annu. Conf. of Illum. Engng. Inst. Jpn. (1982) no. 55

(2) Sato, Kaneko, Kobayashi, Otaki: Development of Recessed HID Downlight Utilizing Prism Cover having Squared Illuminance Distribution, preprints of Annu. Conf. of Illum. Engng. Inst. Jpn. (1983) no. 62

(3) Nakase, Sato, Hamaguchi, Muraki: Power-Saving Type Road Signboard, Toshiba Review (1982) 713-716

(4) Sato, Kaneko, Kobayashi: HID Downlight Sofrax having Squared Illuminance Distribution, Toshiba Review (1984) 207-210

(5) Yamada, Kaneko: Development of Fluorescent Lighting Fixture Utilizing Prism Panel Having Twin Beam Luminous Intensity Distribution, preprints of Annu. Conf. of Illum. Engng. Inst. Jpn. (1984) no. 29

(6) Mizushima: Transparent Cover for Lighting Fixture Having Continuous Quadrangular Pyramid Pattern, JP Utility Model Sho 48-29752 (1973)

(7) Richard Carol: Illumination Panel, JP Patent Application Sho 49-83281 (1974)

(8) Shimizu, Nomura: Optical Property Analysis of Prism Panel and Reflecting Mirror, Tech. J. of Matsushuta, no. 22 (1981) 17-23

(9) Sakaguchi, Funabashi, Murakami, Otsuka: Development of Luminous Intensity Controlling Panel for Diffusing Light Source, Elec. Engng. Related Gen. Symp. (Kansai) (1985) G311

(10) Kurosawa: Photometric Theory of Floodlight III, J. Illum. Engng. Inst. Jpn. (1952) 150-156

(11) Sato, Matsushita: Application of Computer in Optical Design of Lighting Fixture, J. Illum. Engng. Inst. Jpn. (1982) 550-554

Received January 21, 1991; accepted February 11, 1991. 